\title{
Digital Testing Device for Active Range of Motion of Finger Joints Utilizing Artificial Neural Network
}

\author{
Huu-Hieu Quang \\ Department of Electrical and Mechanical Engineering, Graduate School of Engineering \\ Nagoya Institute of Technology, Gokiso-cho, Showa-ku, Nagoya, Aichi 466-8555, Japan \\ Yoshifumi Morita \\ Department of Electrical and Mechanical Engineering, Graduate School of Engineering \\ Nagoya Institute of Technology, Gokiso-cho, Showa-ku, Nagoya, Aichi 466-8555, Japan
}

\author{
Makoto Takekawa \\ everfine, LLC, Japan \\ Email:31513006@stn.nitech.ac.jp,morita@nitech.ac.jp, \\ makoto.takekawa@gmail.com \\ www.nitech.ac.jp
}

\begin{abstract}
This paper proposed a digital goniometer device for measuring the active range of motion (A-ROM) of finger joints by artificial neural network (ANN). A therapist typically measures the A-ROM by a conventional goniometer. However, it consumes a significant amount of time, and imposes a burden on patients. Therefore, we develop a digital goniometer using ANN to overcome these problems. The effectiveness of the proposed device is verified based on the evaluation error on the third joint of the middle finger.

Keywords: Digital goniometer device, Active range of motion, Artificial network, Conventional goniometer.
\end{abstract}

\section{Introduction}

The range of motion (ROM) is an essential part in clinical practices when assessing the effectiveness of therapeutics. A therapist typically measures the ROM by conventional, as shown in Fig. 1, or digital goniometers. ${ }^{1,2}$

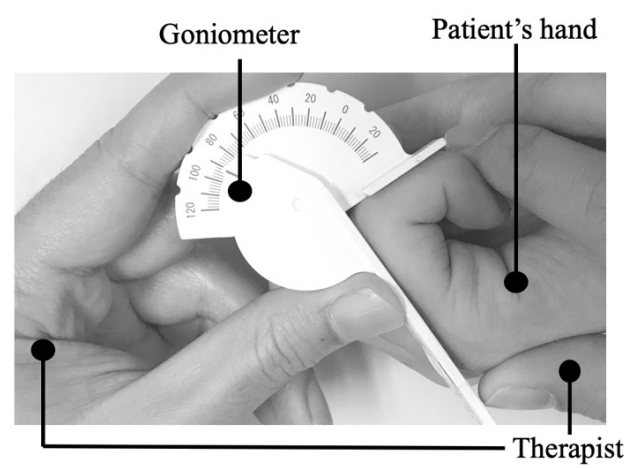

Fig. 1. A-ROM test with a conventional goniometer.

However, he/she cannot carry out the measurement in a short time by these goniometers. Accordingly, several measuring methods were proposed by extant studies. ${ }^{3,4} \mathrm{~A}$ VICON system using markers placed on each joint of the fingers were proposed in order to compute the joint angles. In addition, a non-invasive automatic testing device was developed to boost the measurement time and accuracy. Nevertheless, the developed devices cannot measure the ROM precisely in a short time, and the response of the systems during operation is poor. In addition, a non-invasive automatic testing device was developed to boost the measurement time and accuracy. Nevertheless, the developed devices cannot measure the ROM precisely in a short time, and the response of the systems during operation is poor.

In this study, a digital testing goniometer is developed to overcome these problems. A data collection model is proposed to collect training dataset of the finger joints. Subsequently, an ANN model is utilized to validate the dataset. Hence, it can facilely compute the finger joint angles. From the viewpoint of improving measurement accuracy, the effectiveness of the proposed Digital testing goniometer is evaluated on the third joint of the middle finger in comparison with the conventional goniometer.

(C) The 2021 International Conference on Artificial Life and Robotics (ICAROB2021), January 21 to 24, 2021 


\section{A-ROM Testing Method and Device}

\subsection{Outline of digital testing device}

The active range of motion (A-ROM) is the angle when a patient bends or extends his/her own joints as much as possible. Meanwhile the passive range of motion (PROM) is the joint angles when the therapist bends or extends the patient's joints as much as possible. Figure 2 shows an overview of digital goniometer device where the depth camera (Intel RealSense Depth Camera SR300) was connected to the PC via the USB gate. The depth image of depth camera has $640 \times 480$ pixel resolution, and a trigger frequency of $30 \mathrm{~Hz}$.

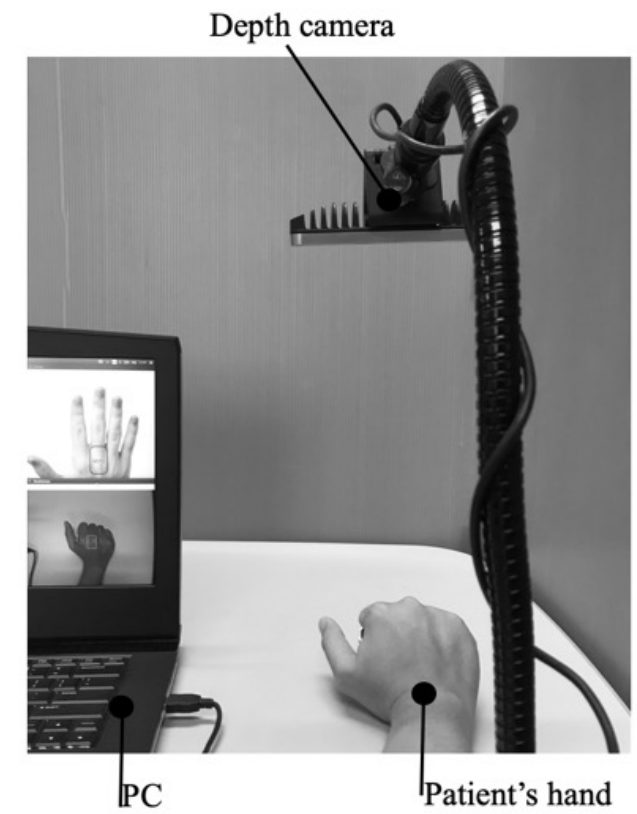

Fig. 2. Digital testing goniometer.

\subsection{A-ROM testing method}

In this study, the metacarpophalangeal joint (MP) of the middle finger is considered. The MP joint is the third joint and the joint between two bones, namely the metacarpal and the wrist bones. The range of motion of MP joints in healthy people is 0 to $90 \mathrm{deg}$ (flexion) and 0 to $45 \mathrm{deg}$ (extension). Let the fully extended position be the neutral position of $0 \mathrm{deg}$. During A-ROM testing, the therapist moves the depth camera towards to the patient's hand so that the middle finger is into the three-green boxes, as shown in Fig. 3(a). The three green boxes are overlaid on the image of RGB camera. The interface screen shows the taken images on both RGB and depth modes simultaneously, as shown in Fig. 3.

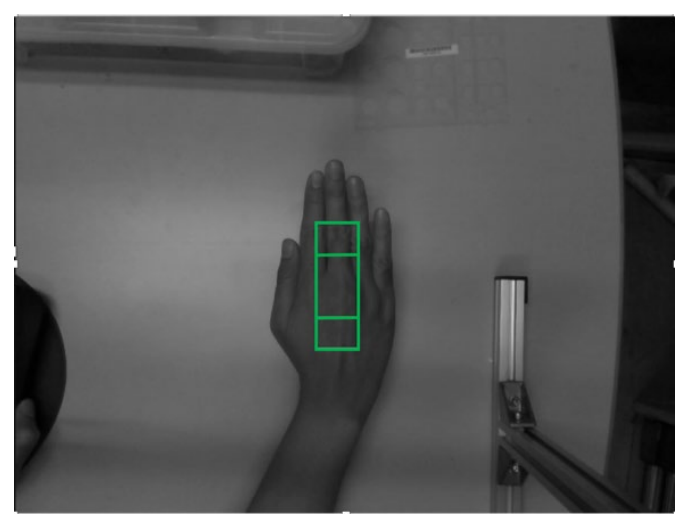

(a) RGB image

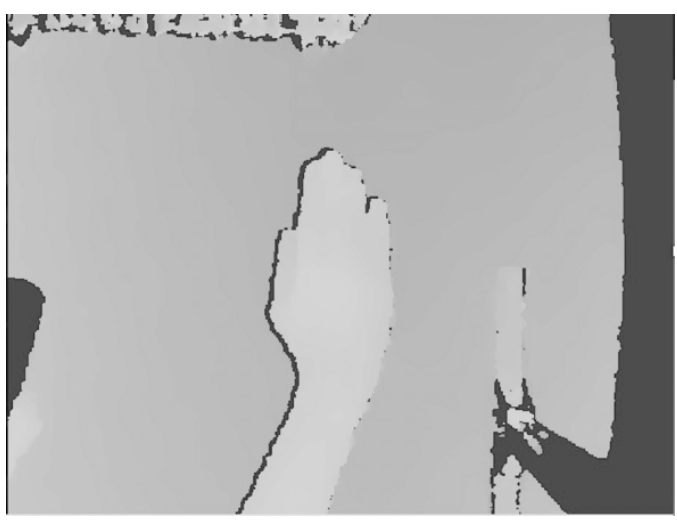

(b) Depth image

Fig. 3. Interface of system.

\section{A-ROM Measurement by ANN Method}

\subsection{Principle}

The points of the proximal interphalangeal, metacarpophalangeal joints of the middle finger and the center point of wrist bones are denoted by PIP3, MP3, and $\mathrm{W}$, respectively, as shown in Fig. 4. The XYZ-axes of the depth camera coordinate system are shown in Fig. 4. The three-dimensional position coordinates of three points are measured with the depth camera. The unit of $\mathrm{X}$ - and $\mathrm{Y}$-axes is pixel. The unit of $\mathrm{Z}$-axis is meter. The coordinate of each joint is determined by RGB image and Depth image data. It should be noted that the units are different. Subsequently, the joint angle is predicted by using an ANN model since there is a linear regression relevance between the joint's position coordinate and joint angle. 


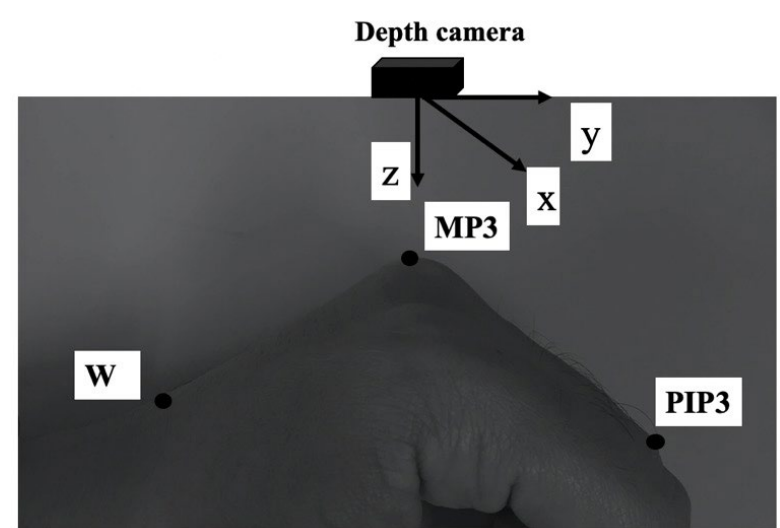

Fig. 4. Positions of the phalangeal joints of the middle finger.

\subsection{Data collection}

We constructed an ANN model from three-dimensional position coordinates of W, MP3 and PIP3 joints to the MP joint angle. We created data collection model for finger joints position detection by using Hand Keypoints, which was a well-known open-source model in the field of image processing for high predictive accuracy of fingers joints recognition. ${ }^{5}$ Using the image data acquired by the RGB camera and the position of three points determined by the Hand Keypoints, the two-dimension position coordinates of three joints are obtained in pixel units. Moreover, using the depth data acquired by the depth camera, the depth coordinates corresponding the two-dimensional position coordinates of three points are obtained in meters.

Moreover, three green boxes are set so that the therapist can easily locate the patient's hand, as shown Fig. 5. The overall size of three boxes is $40 \times 120$ pixel, the size of each green box is $40 \times 30$ pixel, $40 \times 60$ pixel, and $40 \times 30$ pixel from the top to the bottom, respectively. The position of PIP3, MP3, and W joints are marked by red, blue, and green points, and are denoted by I, J, and $\mathrm{K}$ points in Fig. 5, respectively. The MP joint angle was determined by the three points. The input dataset consists of the coordinates of these points. In addition, these points are features of ANN model.

$\mathrm{I}_{\mathrm{i}}=\left(\mathrm{x}_{\mathrm{Ii}}, \mathrm{y}_{\mathrm{Ii}}, \mathrm{z}_{\mathrm{Ii}}\right), \mathrm{J}_{\mathrm{i}}=\left(\mathrm{x}_{\mathrm{Ji}}, \mathrm{y}_{\mathrm{Ji}}, \mathrm{z}_{\mathrm{Ji}}\right), \mathrm{K}_{\mathrm{i}}=\left(\mathrm{x}_{\mathrm{Ki}}, \mathrm{y}_{\mathrm{Ki}}, \mathrm{z}_{\mathrm{Ki}}\right)$ $(\mathrm{i}=1,2, \ldots, \mathrm{n}), \mathrm{n}$ is the number of data contained in the dataset. When the data is acquired for constructing and evaluating a ANN model, the subject moved the hand until $\mathrm{I}, \mathrm{J}$ and $\mathrm{K}$ points are inside the corresponding green boxes.

The output of the dataset is the MP joint angle, which was measured with a conventional goniometer. When measuring the joints of the fingers, the joints were fixed by sandwiching clay between the joints so that the joint angle of the fingers did not change. Table 1 shows the MP joint angle measured with the conventional goniometer, which are used for constructing a ANN model and evaluating the accuracy.

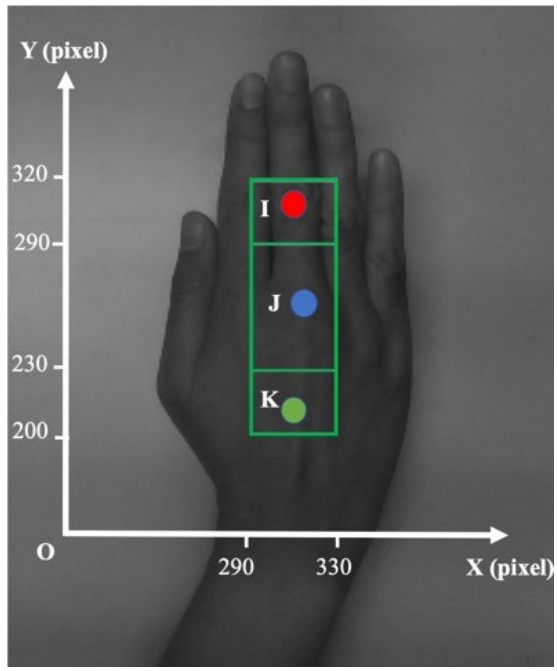

Fig. 5. Processing image to collect data.

Table 1. Measured values of MP joint angle with the conventional goniometer.

\begin{tabular}{lcccccc}
\hline Pose No. & 1 & 2 & 3 & 4 & 5 & 6 \\
\hline Angle[deg] & 38 & 50 & 64 & 68 & 73 & 80 \\
\hline
\end{tabular}

\subsection{Artificial Neural Network}

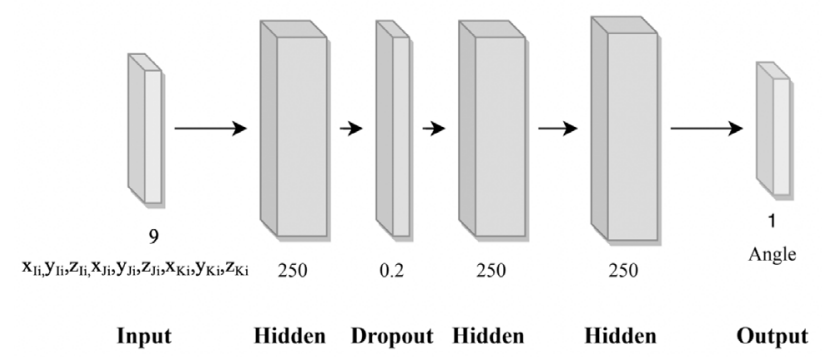

Fig. 6. Artificial neural network architecture.

The principle of the method is creating a model by ANN. ${ }^{6}$ The model is determined based on the linear relationship between the joint position and joint angle of sample 
dataset. Subsequently the model is used to predict the joint angle based on the joint position. We used ANN model for regression to predict angle value. The ANN model used Rectified Linear Unit (ReLu) activation function in both layers. The loss function utilized for regression was Mean Squared Error (MSE). In addition, Adam was chosen as the optimizer function. The architecture of the artificial neural network is shown in Fig. 6.

ANN model was configured of three middle layers of 250 neurons represented by ReLu activation function. In addition, a dropout layer was added as a regularization technique in regression network. The dropout layer randomly drops some of the inputs to a layer is 0.2 .

\section{Evaluation of Measurement Predicted Accuracy}

The evaluation of measurement accuracy in A-ROM testing was conducted to investigate the effectiveness of the proposed digital goniometer device. For this purpose, $75 \%$ of the dataset was randomly selected for constructing the ANN model and the remaining 25\% was assigned for validation. The effectiveness of the proposed ANN model was evaluated base on the $25 \%$ dataset. Figure 7 shows the error between the measured angle with the conventional goniometer and the predicted angle by the proposed testing device. The horizontal axis denotes the trials of 1447 . Figure 8 shows the cumulative histogram of the errors. It was found that the errors of $98.69 \%$ and $99.52 \%$ trials were less than $2 \mathrm{deg}$ and $5 \mathrm{deg}$, respectively.

\section{Conclusion}

In order to solve the problems faced when using a conventional goniometer, we proposed a digital goniometer device for measuring the active range of motion of the MP joint of the middle finger. We constructed an estimation model of the finger joint by using RGB and depth data of the finger joint, the Hand Keypoints, and the ANN model. The estimation accuracy of the MP joint of the middle finger was less than $2 \mathrm{deg}$. The effectiveness of the proposed device was demonstrated.

In our further study, we plan to extend the proposed method so that it can be applied to 12 finger joints. Moreover, we plan to estimate the data collection model to remove outlier for increase high performance of the ANN model as well as reduce error value.

\section{References}

1. Goniometer for fingers, SAKAI Medical Co.,Ltd., https://www.sakaimed.co.jp/measurement analysis/huma n-body-measurement/hand-goniometer/ (In Japanese).

2. easyangle, Ito Co., Ltd., http://www.medical.itolator.co.jp/product/easyangle/ (In Japanese).

3. J. R. Cook, N. A. Baker, R. Cham, E. Hale and M. S. Redfern, Measurements of Wrist and Finger Postures: A Comparison of Goniometric and Motion Capture Techniques, Journal of Applied Biomechanics, 23, 2007, pp.70-78.

4. Reissner L, Fischer G, List R, Taylor WR, Giovanoli P, Calcagni M, Minimal detectable difference of the finger and wrist range of motion: comparison of goniometry and 3D motion analysis, J. Orthop Surg Res, 14(1), 2019.

5. Simon, Tomas \& Joo, Hanbyul \& Matthews, Iain \& Sheikh, Yaser. Hand Keypoint Detection in Single Images using Multiview Bootstrapping, Cornell University, USA, 2017.

6. Lau, E.T., Sun, L. \& Yang, Q. Modelling, prediction and classification of student academic performance using artificial neural networks. SN Appl. Sci. 1, 982, 2019.

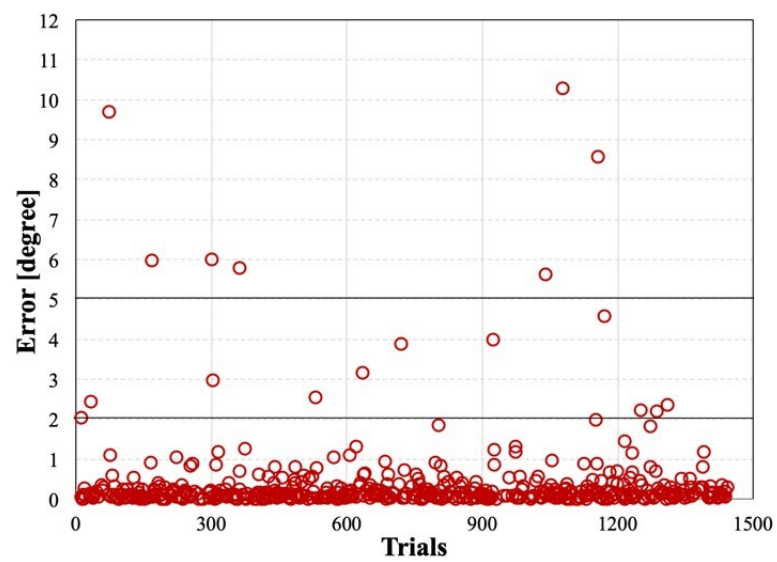

Fig. 7. Distribution of the errors of predicted angles.

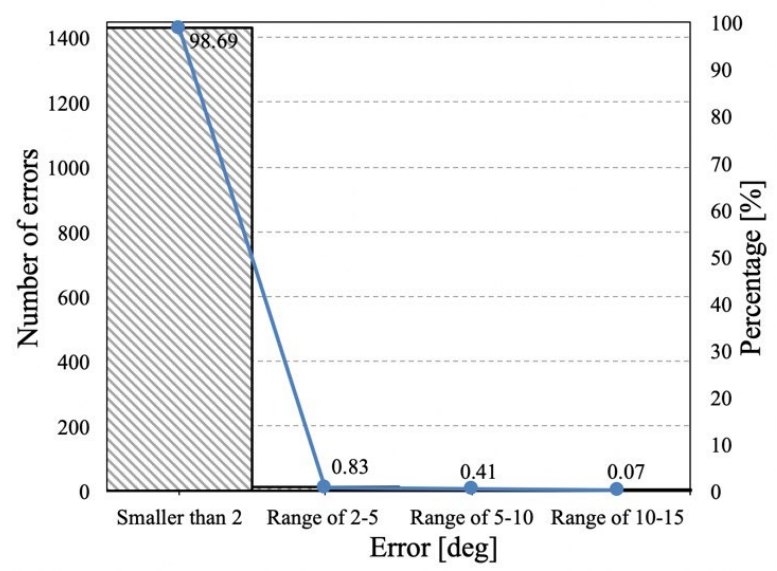

Fig. 8. Histogram of the errors of predicted angles. 